\title{
DETERMINANTAL RANK AND FLAT MODULES ${ }^{1}$
}

\author{
S. H. COX, JR.
}

1. By ring we mean commutative ring with identity. Module means unitary module. In this paper we use some results on determinantal rank to prove the following proposition: A finitely generated $R$-module $M$ is projective if and only if $M$ is flat and there is an exact sequence $0 \rightarrow M \rightarrow N \rightarrow L$ of $R$-modules such that $N$ and $L$ are projective (Theorem 2.9). A corollary is that a finitely generated $R$ module $M$ is projective if and only if $M$ is flat, reflexive and $M^{*}=\operatorname{Hom}_{R}(M, R)$ is of finite presentation. In $\$ 3$, we give an example of a cyclic ideal $M$ in a ring $R$ such that $M$ is flat and reflexive, $M^{*}$ is cyclic, but $M$ is not projective.

We use f.g. in place of finitely generated and morphism instead of $R$-homomorphism. The set of prime ideals of a ring $R$ is denoted $\operatorname{Spec}(R) . N$ denotes the set of nonnegative integers. If $S \subset N$ is unbounded, we write $\sup (S)=\infty$.

2. Let $u: M \rightarrow N$ be a morphism of $R$-modules. We define $\operatorname{rk}(u)$, the rank of $u$, by $\operatorname{rk}(u)=\sup \left\{n \in N ; \Lambda^{n} u \neq 0\right\}$ where $\Lambda^{n}$ denotes $n$th exterior power. We also define $\operatorname{dim}(M)=\operatorname{rk}\left(1_{M}\right)$. When $M$ and $N$ are f.g. free $R$-modules, $\mathrm{rk}(u)$ is also the determinantal rank of a matrix corresponding to $u$ and $\operatorname{dim}(M)$ is the cardinality of a basis of $M$. When $M$ and $N$ are free we denote by $D(u, p)$ the ideal generated by the $p$-minors of a matrix corresponding to $u$. The ideals $\{D(u, p) ; p \in N\}$ are the Fitting invariants of $\operatorname{Coker}(u)$ [3]. If $S$ is a multiplicative system in $R$, then $\mathrm{rk}\left(u_{S}\right)$ is the rank of $u_{S}$ as an $R_{S}$-morphism.

The following result from [2, p. 98, Exercise 3] will be used several times.

2.1. Lemma. Let $M$ and $N$ be f.g. free $R$-modules of dimensions $m$ and $n$ respectively. Then a morphism $u: M \rightarrow N$ is a monomorphism if and only if $m \leqq n$ and $\operatorname{Ann}(D(u, m))=0$. (In that case $\operatorname{rk}(u)=m$.)

2.2. Lemma. Let $u: M \rightarrow N$ be a morphism of $R$-modules. Let $S$ and $T$ be multiplicative systems in $R$ such that $S \leqq T$ (i.e. $R \rightarrow R_{T}$ factors through $\left.R \rightarrow R_{S}\right)$. Then

(i) $\operatorname{rk}\left(u_{T}\right) \leqq \operatorname{rk}\left(u_{S}\right)$,

Received by the editors June 10, 1968.

1 The contents of this paper form part of the author's 1968 Louisiana State University Ph.D. Dissertation. I wish to thank Professor R. L. Pendleton for his assistance in serving as my faculty advisor, and for his advice during the preparation of this paper. 
(ii) if $M$ is f.g., then $\exists f \in S \cdot \exists \cdot \operatorname{rk}\left(u_{f}\right)=\operatorname{rk}\left(u_{S}\right)$,

(iii) if $L \dot{\rightarrow} M$ is an $R$-morphism, then $\mathrm{rk}(u v) \leqq \min \{\operatorname{rk}(u), \operatorname{rk}(v)\}$,

(iv) $\mathrm{rk}(u)=\sup \left\{\operatorname{rk}\left(u_{p}\right) ; p \in \operatorname{Spec}(R)\right\}$.

The proof of 2.2 is straightforward.

2.3. Proposition. Let $v: M \rightarrow N$ and $u: N \rightarrow L$ be morphisms of $f . g$. free $R$-modules such that Image $(v) \supseteq \operatorname{Kernel}(u)$. Then either $\operatorname{dim}(N)$ $\leqq \mathrm{rk}(u)+\operatorname{dim}(M)$ or $D(v, \operatorname{dim}(M)) \subseteq \sqrt{ } 0$.

Proof. Let $m=\operatorname{dim}(M), n=\operatorname{dim}(N)$. Suppose that $D(v, m) \Phi \sqrt{ } 0$. Since we wish to show that $n \leqq \mathrm{rk}(u)+m$, we may assume $m \leqq n$. As $D(v, m) \Phi \sqrt{ } 0, \exists p \in \operatorname{Spec}(R) \cdot \ni \cdot D(v, m) \Phi p$. Then it is easy to see that $D\left(v_{p}, m\right)=(D(v, m))_{p}=R_{p}$. Thus by $\left[2\right.$, p. 98, Exercise 5] $\left(v_{p}\right)^{*}:\left(N_{p}\right)^{*}$ $\rightarrow\left(M_{p}\right)^{*}$ is an epimorphism. Then by $\left[1\right.$, p. 108, Proposition 6] $v_{p}$ is a monomorphism onto a direct summand of $N_{p}$. Let $v_{p}\left(M_{p}\right) \oplus H=N_{p}$ and let $c: H \rightarrow N_{p}$ be the canonical inclusion. Evidently $H$ is a free $R_{p}$-module of dimension $n-m$. Since Image $\left(v_{p}\right) \supseteq \operatorname{Kernel}\left(u_{p}\right), u_{p} c$ is a monomorphism. Hence $\operatorname{rk}\left(u_{p} c\right)=n-m$. By 2.2(i) and 2.2(iii), $\operatorname{rk}(u) \geqq n-m$.

2.4. Corollary. If $\boldsymbol{v}$ in 2.3 is a monomorphism, then $\operatorname{dim}(N)$ $\leqq \mathrm{rk}(u)+\operatorname{dim}(M)$.

Proof. Let $D=D(v, \operatorname{dim}(M))$. By $2.1, \operatorname{Ann}(D)=0$. Thus, because $D$ is f.g., $D \Phi \sqrt{ } 0$. Hence, by $2.3, \operatorname{dim}(N) \leqq \operatorname{rk}(u)+\operatorname{dim}(M)$.

2.5. Corollary. Let $u: N \rightarrow L$ be a morphism of f.g. flat R-modules. Let $M$ be a f.g. flat submodule of $N$ such that $M \supseteq \operatorname{Kernel}(u)$. Then $\operatorname{dim}(N) \leqq \mathrm{rk}(u)+\operatorname{dim}(M)$.

Proof. Let $p \in \operatorname{Spec}(R)$. By 2.4 and 2.2(i),

$$
\operatorname{dim}\left(N_{p}\right) \leqq \operatorname{rk}\left(u_{p}\right)+\operatorname{dim}\left(M_{p}\right) \leqq \operatorname{rk}(u)+\operatorname{dim}(M) .
$$

Hence, by 2.2 (iv), $\operatorname{dim}(N) \leqq \operatorname{rk}(u)+\operatorname{dim}(M)$.

2.6. Proposition. Let $v: E \rightarrow F, u: F \rightarrow G$ be morphisms of f.g. free $R$-modules, $n=\operatorname{dim}(F)$. If $0 \leqq p, q \leqq n$ are integers such that $p+q>n$, and if $u v=0$, then $D(v, q) D(u, p)=0$.

Proof. We may assume that $E=F=G$. Let $e_{1}, \cdots, e_{n}$ be a basis of $E$. Let $U=\left(u_{i j}\right)$ and $V=\left(v_{i j}\right)$ be the matrices of $u$ and $v$, respectively, relative to $e_{1}, \cdots, e_{n}$. We use the notation of [2]. We must show that if $H, K, S, T \subseteq[1, n] \cdot \ni \cdot|H|=|K|=p$ and $|S|=|T|=q$, then $V_{S, T} U_{H, K}=0$. Let $H, K, S, T \subseteq[1, n]$ with $|H|=|K|=p$ and $|S|=|T|=q$. We will construct an endomorphism $w$ of $E \cdot \ni \cdot \operatorname{det}(w)$ 
$=t V_{S, T} U_{H, K}$ for some non-zero-divisor $t$ of $R$. Then we will show $\operatorname{det}(w)=0$. For $L \subseteq[1, n], \pi_{L}: E \rightarrow E$ is the projection defined by $\pi_{L}\left(e_{i}\right)=e_{i}$ if $i \in L$ and $\pi_{L}\left(e_{i}\right)=0$ if $i \in L^{\prime}$. Select one-to-one correspondences $\sigma: K^{\prime} \rightarrow H^{\prime}$ and $\tau: T^{\prime} \rightarrow S^{\prime}$. Let $\alpha=\sigma^{-1}$ and $\beta=\tau^{-1}$. Define $f_{\sigma}: E \rightarrow E$ by $f_{\sigma}\left(e_{i}\right)=e_{\sigma(i)}$ if $i \in K^{\prime}$, and $f_{\sigma}\left(e_{i}\right)=0$ if $i \in K$. Define $f_{\tau}, f_{\alpha}$ and $f_{\beta}$ similarly. It is easy to check that

(i) $\pi_{S}+\pi_{S^{\prime}}=1_{E}=\pi_{K}+\pi_{K^{\prime}}$,

(ii) $f_{\tau} f_{\beta}=\pi_{S^{\prime}}, f_{\alpha} f_{\sigma}=\pi_{K^{\prime}}$,

(iii) $\pi_{T} f_{\beta}=0=f_{\alpha} \pi_{H}=\pi_{H} f_{\sigma}=\pi_{S} f_{r}$,

(iv) $f_{\beta} v \pi_{T}+1_{E}$ and $\pi_{H} u f_{\alpha}+1_{E}$ are monomorphisms.

Now let $w=\left(\pi_{H} u+f_{\sigma}\right)\left(v \pi_{T}+f_{\tau}\right)$. Using (i)-(iii), we get that $\pi_{H} u+f_{\sigma}$ $=\left(\pi_{H} u f_{\alpha}+1_{E}\right)\left(\pi_{H} u \pi_{K}+f_{\sigma}\right)$. By 2.1, $t_{1}=\operatorname{det}\left(\pi_{H} u f_{\alpha}+1_{E}\right)$ is a non-zerodivisor of $R$. By Laplace's Expansion, $\operatorname{det}\left(\pi_{H} u \pi_{K}+f_{\sigma}\right)= \pm U_{H, K}$. Hence, $\operatorname{det}\left(\pi_{H} u+f_{\sigma}\right)= \pm t_{1} U_{H, K}$. Similarly, $\operatorname{det}\left(v \pi_{T}+f_{\tau}\right)= \pm t_{2} V_{S, T}$ where $t_{2}=\operatorname{det}\left(f_{\beta} v \pi_{T}+1_{E}\right)$ is a non-zero-divisor of $R$. Hence, $\operatorname{det}(w)$ $=t V_{S, T} U_{H, K}$ where $t$ is a non-zero-divisor of $R$. Now let $W$ be the matrix of $w$ relative to $e_{1}, \cdots, e_{n}$. We have

$$
\operatorname{det}(W)=\rho_{T, T^{\prime}} \Sigma_{L} \rho_{L, L^{\prime}} W_{L, T} W_{L^{\prime}, T^{\prime}}
$$

by Laplace's Expansion. Let $L \subseteq[1, n] \cdot \ni \cdot|L|=|T|=q$. Then $L \cap H \neq \varnothing$ since $|H|=p$ and $p+q>n$. Choose $j \in L \cap H$. Then $\pi_{\{j\}} w \pi_{T}=0$. Hence, the $j$ th row of the $q \times q$ submatrix of $W$ determined by rows in $L$ and columns in $T$ is zero. Therefore $W_{L, T}=0$, and $\operatorname{det}(W)=0$. Finally, $U_{H, K} V_{S, T}=0$.

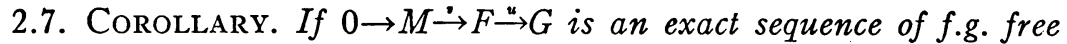
$R$-modules, then $\operatorname{dim}(M)+\mathrm{rk}(u)=\operatorname{dim}(F)$.

Proof. By 2.4, $\operatorname{dim}(F) \leqq \operatorname{dim}(M)+\mathrm{rk}(u)$. By 2.6, $D(u, n-m+1)$ $\cdot D(v, m)=0$ where $n=\operatorname{dim}(F)$ and $m=\operatorname{dim}(M)$. By 2.1, Ann $(D(v, m))$ $=0$. So $D(u, n-m+1)=0$. Therefore, $\wedge^{n-m+1} u=0$, i.e., $\operatorname{rk}(u) \leqq n-m$.

2.8. Proposition. If $M \stackrel{\rightarrow}{\rightarrow} \stackrel{\leftrightarrow}{\rightarrow} G$ is an exact sequence of $f . g$. free $R$-modules such that $\mathrm{rk}(u)+\operatorname{dim}(M)=\operatorname{dim}(F)$ and if Image $(v)$ is flat, then $v$ is a monomorphism.

Proof. Let $p \in \operatorname{Spec}(R)$. Since $v(M)$ is flat, $v_{p}\left(M_{p}\right)$ is a free $R_{p^{-}}$ module. By 2.7, $\operatorname{dim}\left(v_{p}\left(M_{p}\right)\right)+\operatorname{rk}\left(u_{p}\right)=\operatorname{dim}\left(F_{p}\right)$. By $2.2(\mathrm{i}), \operatorname{rk}\left(u_{p}\right)$ $\leqq \mathrm{rk}(u)$. Thus,

$$
\begin{aligned}
\operatorname{dim}\left(M_{p}\right) \geqq \operatorname{dim}\left(v_{p}\left(M_{p}\right)\right) & =\operatorname{dim}\left(F_{p}\right)-\operatorname{rk}\left(u_{p}\right) \geqq \operatorname{dim}\left(F_{p}\right)-\operatorname{rk}(u) \\
& =\operatorname{dim}(F)-\operatorname{rk}(u)=\operatorname{dim}(M)=\operatorname{dim}\left(M_{p}\right) .
\end{aligned}
$$

So $M_{p} \rightarrow v_{p}\left(M_{p}\right)$ is an epimorphism of free $R_{p}$-modules of the same 
dimension. Hence, $M_{p} \rightarrow v_{p}\left(M_{p}\right)$ is an isomorphism. Therefore $v_{p}$ is a monomorphism, $\forall p \in \operatorname{Spec}(R)$. Therefore $v$ is a monomorphism.

2.9. Theorem. A finitely generated $R$-module $M$ is projective if and only if $M$ is flat and there is an exact sequence $0 \rightarrow M \stackrel{\rightarrow}{\rightarrow} \stackrel{\leftrightarrow}{\rightarrow} G$ with $F$ and $G$ projective $R$-modules.

Proof. If $M$ is projective, then certainly $M$ is flat and such a sequence exists. Conversely, it is not hard to see that we may assume that $F$ and $G$ are free and f.g. Now let $p \in \operatorname{Spec}(R)$. By 2.2.(ii), $\exists f^{\prime} \in R \backslash p \cdot \exists \cdot \operatorname{rk}\left(u_{f^{\prime}}\right)=\operatorname{rk}\left(u_{p}\right)$. Let $m=\operatorname{dim}\left(M_{p}\right)$. By $2.7, m+\operatorname{rk}\left(u_{p}\right)$ $=\operatorname{dim}\left(F_{p}\right)$. Let $E$ be a free $R$-module of dimension $m$. There is an

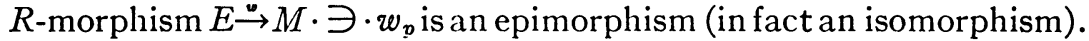
By [2, p. 136, Proposition 2], $\exists f^{\prime \prime} \in R \backslash p \cdot \ni \cdot w_{f^{\prime \prime}}$ is an epimorphism. Let $f=f^{\prime} f^{\prime \prime}$. Then $\operatorname{rk}\left(u_{f}\right)=\operatorname{rk}\left(u_{p}\right)$, $w_{f}$ is an epimorphism, and $f \in R \backslash p$. The sequence $E_{f} \rightarrow F_{f} \rightarrow G_{f}$ is an exact sequence of f.g. free $R_{f}$-modules; Image $\left(w_{f}\right)=M_{f}$ is a flat $R_{f}$-module;

$$
\operatorname{rk}\left(u_{f}\right)+\operatorname{dim}\left(E_{f}\right)=\operatorname{rk}\left(u_{p}\right)+\operatorname{dim}\left(M_{p}\right)=\operatorname{dim}\left(F_{p}\right)=\operatorname{dim}\left(F_{f}\right) .
$$

Hence, by $2.8, w_{f}$ is a monomorphism. Therefore, $M_{f}=\operatorname{Image}\left(w_{f}\right)$ is a free $R_{f}$-module. We have shown that $\forall p \in \operatorname{Spec}(R) \exists f \in R \backslash p \cdot \exists \cdot M_{f}$ is a free $R_{f}$-module. Thus $M$ is projective by $[1, \mathrm{p} .138$, Theorem 1$]$.

2.10. Corollary. A finitely generated $R$-module $M$ is projective if and only if $M$ is flat, reflexive and $M^{*}$ is of finite presentation.

Proof. The necessity is well known. For the converse, let $f: M$ $\rightarrow M^{* *}$ be the canonical morphism.

Since $M^{*}$ is of finite presentation, there is an exact sequence $E \stackrel{\bullet}{\rightarrow} F \stackrel{h}{\rightarrow} M^{*} \rightarrow 0$ with $E$ and $F$ f.g. free $R$-modules. Hence, $0 \rightarrow M^{h * 4} \rightarrow F^{*}$ $\stackrel{\Delta^{*}}{\rightarrow} E^{*}$ is exact and $F^{*}$ and $E^{*}$ are free. By $2.9, M$ is projective.

3. Let $S$ be a ring which admits a commutative $S$-algebra $A \neq 0$ satisfying

(1) there is a non-zero-divisor $t$ of $S$ such that $t A=0$,

(2) $\forall a \in A \exists b \in A \cdot \ni \cdot b a=a$,

(3) $A$ has no multiplicative identity.

Let $R=S \times A$ with the usual coordinate addition and multiplication defined by $(s, a)(r, b)=(s r, r a+s b+a b)$. Fix a non-zero-divisor $t$ of $S$ such that $t A=0$. Let $r=(t, 0)$ and $M=R r$. Denote the exact sequence $0 \rightarrow \mathrm{Ann}_{R}(M) \rightarrow R \rightarrow M \rightarrow 0$ by $(E) . M$ is flat: it is sufficient to show $x \in \mathrm{Ann}_{R}(M) \Rightarrow \exists y \in \mathrm{Ann}_{R}(M) \cdot \ni \cdot y x=x[1, \mathrm{p} .65$, Exercise 23]. Let $x \in \operatorname{Ann}_{R}(M)$. Write $x=(s, a)$. Since $x r=0$ and $t$ is a non-zero-divisor, $s=0$. By (2), $\exists b \in A \cdot \exists \cdot b a=a$. Let $y=(0, b)$. Since $t A=0, y r=0$ so 
$y \in \operatorname{Ann}_{R}(M)$. Also $y x=(0, b)(0, a)=(0, b a)=(0, a)=x$. Thus $M$ is flat. $M$ is not projective: if $M$ is projective, then $(E)$ splits. Hence $\operatorname{Ann}_{R}(M)$ is generated by an idempotent $e$ of $R$. Since $A \neq 0, e \neq 0$. Write $e=(0, u)$. Let $c \in A$. Then $(0, c) r=0$ so $(0, c) \in \operatorname{Ann}_{R}(M)$. Therefore, $(0, c)=e(0, c)=(0, u)(0, c)=(0, u c)$. That is $u c=c, \forall c \in A$ contradicting (3). Thus $M$ is a cyclic flat nonprojective ideal of $R$.

Now consider the following choice for $S$ and $A . S$ is the ring of integers. $I$ is an infinite set and $A$ is the set of functions $f: I \rightarrow S /(2)$ such that $f(i)=0$ for all but finitely many $i \in I$. With pointwise operations, $A$ is an $S$-algebra satisfying (1)-(3) with $t=2$ in (1). Thus $M=R(2,0)$ is flat but not projective. It is easy to see that in this case we have $M=\operatorname{Ann}_{R}\left(\operatorname{Ann}_{R}(M)\right)$. It follows that $M^{*}$ is cyclic and $M$ is reflexive. Hence, $M$ is a cyclic flat reflexive nonprojective ideal with $M^{*}$ cyclic. This shows that the hypothesis " $M^{*}$ is of finite presentation" in 2.10 cannot be replaced by " $M^{*}$ is f.g."

\section{REFERENCES}

1. N. Bourbaki, Éléments de mathématique. Algèbre commutative, Chapters 1 and 2, Hermann, Paris, 1961.

2. ——_ Éléments de mathématique. Algèbre, Chapter 3, Hermann, Paris, 1958.

3. H. Fitting, Die Determinantenideale eines Moduls, Jber. Deutsch. Math.Verein. 46 (1936), 195-228.

Louisiana State University 